\title{
Sudden death due to acute adrenal crisis
}

\author{
Cristian Palmiere ${ }^{1}$
}

Accepted: 29 April 2015/Published online: 29 May 2015

(C) Springer Science+Business Media New York 2015

We read the article by Govi et al. [1] with great interest. The diagnosis of acute adrenocortical insufficiency as a cause of death is particularly challenging in the forensic setting [2-4]. What is particularly noteworthy in the case described by the authors is the statement that the recurrent respiratory tract infections, liquid fecal staining, and an empty gastrointestinal tract were distinctly indicative of adrenal crisis. Although the information pertaining to recurrent respiratory infections may be of interest in terms of potential, precipitating condition, it does not imply the diagnosis of adrenal crisis as the cause of death in any way. Analogously, diarrhea and an empty gastrointestinal tract may be of interest in terms of both clinical manifestation and triggering condition of adrenal crisis. However, diarrhea alone does not imply the diagnosis of acute adrenocortical insufficiency as the cause of death in any way. In addition, the absence of therapeutic drugs at the death scene may also suggest the sudden discontinuation of steroid therapy as a triggering factor. Lastly, the authors claim the most remarkable finding was the difficulty with which they were able to precisely identify adrenal gland tissue. Again, this statement is not entirely valid since it might provide histological confirmation of adrenal cortical cell atrophy, though not evidence of acute adrenocortical insufficiency.

The authors affirmed that characteristic macroscopic and microscopic findings together with the preexisting medical condition allowed adrenal crisis to be determined as the

Cristian Palmiere

cristian.palmiere@chuv.ch

1 University Center of Legal Medicine (Centre Universitaire Romand de Médecine Légale), Lausanne University Hospital, Chemin de la Vulliette 4, 1000 Lausanne 25, Switzerland cause of death. We believe they have certainly over-interpreted the results of their investigations as the circumstantial, macroscopic and microscopic findings only allow the existence of adrenalitis and adrenal atrophy to be confirmed. The possible role of gastrointestinal disease (and/or steroid treatment withdrawal) as potential, precipitating factor(s) to acute adrenocortical insufficiency could also be postulated, but nothing further. Indeed, the authors have no biochemical investigation results to support the hypothesis of adrenal crisis as the cause of death. Even though this is undoubtedly the most plausible hypothesis, it is not corroborated, in practice, by any laboratory finding.

As far as we are concerned, the case described by the authors emphasizes the necessity of performing exhaustive biochemical analyses and correlating all potentially relevant investigations in autopsy cases involving possibly fatal hormonal disturbances. In the absence of consistent laboratory results, it is unwise to formulate categorical conclusions stating that the cause of death can be manifestly attributed to adrenal crisis beyond a doubt.

\section{References}

1. Govi A, Fersini F, Tsokos M. Sudden death due to acute adrenal crisis. Forensic Sci Med Pathol. 2015. doi:10.1007/s12024-0149647-3.

2. Palmiere C, de Froidmont S, Mangin P, Werner D, Lobrinus JA. Ketoacidosis and adrenocortical insufficiency. J Forensic Sci. 2014;59:1146-52.

3. Clapper A, Nashelsky M, Dailey M. Evaluation of serum cortisol in the postmortem diagnosis of acute adrenal insufficiency. Am J Forensic Med Pathol. 2008;29:181-4.

4. Burke MP, Opeskin K. Adrenocortical insufficiency. Am J Forensic Med Pathol. 1999;20:60-5. 